\title{
Barriers and Strategies: A Review of Access to Affordable Multi-Drug Resistant Tuberculosis Medication in China
}

This article was published in the following Dove Press journal: Infection and Drug Resistance

Yuou Zhang'
Xuan Liu'
Linghe Yang'
Guifang Zhang ${ }^{2}$
Zhaoru Gu (D)
Zhongdan Chen
Jing Sun (D)
'School of Public Health, Chinese
Academy of Medical Sciences and Peking
Union Medical College, Beijing, People's
Republic of China; ${ }^{2}$ Institute of
Geriatrics, Beijing Hospital, Beijing,
People's Republic of China; ${ }^{3}$ Institute of
Cancer, Cancer Hospital Chinese
Academy of Medical Sciences, Beijing,
People's Republic of China; ${ }^{4}$ Hepatitis/
TB/HIV/STI, World Health Organization,
Office of the WHO Representative in
China, Beijing, People's Republic of China

Correspondence: Jing Sun Email sunjing@sph.pumc.edu.cn

\begin{abstract}
This study analyzed the barriers of patient access to affordable MDR-TB medication in China and the reasons behind, and proposed strategies towards removing the barriers based on literature review and key informant interviews. Reasons behind the high financial burden of MDR-TB patients in China are the lack of a coordinated and multi-sourced financing model to secure patients' access to the expensive novel medicines, and the absence of the safety-net for the patients with low ability to pay the costs. Appropriate health insurance benefit packages and provider payment mechanisms, supportive legal framework, coordinated policies as well as incentives for off-label use of evidence-based repurposed medicines are missing. The observations identified key intervention areas including continued efforts to make the novel effective medicines affordable and to strengthen the legislative protection for off-label use of evidence-based medicines; increase incentives for pharmaceutical companies to expand indications of established medicines based on the evidence; implement public initiatives to support the use of repurposed medicines for diseases with major public health significance, and scale up good practices from local pilots to create a coordinated multi-sourced financing model. A comprehensive approach to address the barriers in the full treatment course of MDR-TB and a safety-net for low-ability-to-pay patients are also critical to secure universal access to affordable MDR-TB medication.
\end{abstract}

Keywords: MDR-TB, medication, affordable, financing, safety-net, off-label use, repurposed medicines

\section{Introduction}

Tuberculosis (TB) remains a severe threat to global public health, particularly when patients are infected with multidrug-resistant tuberculosis (MDR-TB). MDR-TB does not respond to at least isoniazid and rifampicin, the two most powerful antiTB medicines, requires longer duration of treatment, daily administration of medicines that are more toxic, more expensive and less effective than those used to treat drug-susceptible TB. Treatment for MDR-TB is more challenging for patients, healthcare providers and the health systems. ${ }^{1,2}$ The world has committed to ending TB by $2030 .^{3}$ Despite the international norm for "free TB care" policies, some countries cover only some diagnostic tests and first-line treatment. ${ }^{4,5}$ TB patients face significant financial burden due to a considerable amount of out-of-pocket (OOP) expenses and loss of incomes. ${ }^{6,7}$

China is the world's second country with the most substantial number of MDRTB patients. As of 2018, this number was $66,000 .^{8}$ The diagnosis rate, the inclusion 
rate of treatment and the success rate of treatment for MDR-TB in China are far below the global average and affected the effective treatment globally., ${ }^{9,10}$ One of the most critical barriers of MDR-TB treatment is that catastrophic cost has increasingly become the major obstacle for people in need of treatment, and patients frequently drop-off from medication due to the financial burden. Interrupted treatment and under-dose led to poor treatment outcomes and increased the risks of resistance and spread of infections. Expansion of a drug-resistance profile with poor adherence to treatment may lead to extensively drugresistance (XDR), which is resistant to at least isoniazid and rifampicin, and to any fluoroquinolone, and to any of the three second-line injectable anti-TB medicines.

\section{Methods}

This study analyzed the barriers of MDR-TB patient access to affordable treatment in China and the reasons behind, proposed short- and long-term strategies towards removing the barriers. The authors reviewed the existing literature, including the crucial papers, news sources, government statistical handbooks and an electronic search of CNKI, Wanfang, CQVIP, Web of Science and PubMed databases with the search terms of "multi-drug resistant tuberculosis" or "MDR-TB" and "affordability"/“finance"/ "financing"/“funding" during the past 10 years in both Chinese and English. A substantial body of evidence available in grey literature was identified as well, including the landmark or highly regarded reports, work suggested by peers and government documents, the national and the World Health Organization (WHO) guidelines and technical reports, the official websites of the national health authority, the national regulatory authority (NRA), the national health security authority and WHO. The authors also drew on insights of the key informants from relevant government agencies at central and local levels and their technical arms, health professionals and pharmaceutical companies through semi-structured qualitative interviews. Detailed information about the key informants and interviews about the barriers for MDR-TB patient access to affordable treatment from the perspectives of healthcare providers, medicines suppliers, drug, health and insurance regulators were listed in Annex 1, 2. No human and animal data were collected, and no interventions were given, so the study is not applicable for the informed consent of the experiment.

\section{Key Barriers of Access to Affordable MDR-TB Medication \\ Gaps in Financing the Expensive Novel Effective Therapies}

Since the 1990s, the national TB control program provided free first-line medications for the diagnosed drug-susceptible TB patients and referred patients with complications to public hospitals in China. This was seen as essential to remove, or at least substantially decrease financial barriers to access standard TB care by the patients in financial hardship. ${ }^{11}$ However, with the development of the increasing numbers of MDR-TB patients, the government funding for TB has been insufficient. In 2019, the funding gap between the reported budget in national strategic plans for $\mathrm{TB}$ and the available funding for this budget from domestic and international donor sources in China reached US\$ 48 million. $^{12}$

The recent health system reforms proposed integration of TB care into specialized infectious diseases hospitals or general hospitals with TB departments. ${ }^{13}$ The reforms shifted the financing of TB from the government budget funded program to the basic health insurance program, which is funded by a combination of the earmarked budget of the central and local governments, the premiums and patient co-payment. Xin et $\mathrm{al}^{14}$ found that integration of the national TB program with the basic health insurance program was an effective strategy to address the challenges of TB by achieving universal population coverage of the basic health insurance in China. However, some of the core medicines for MDR-TB treatment are not covered by the basic health insurance program. ${ }^{15}$

In 2018, the WHO updated guidelines for MDR-TB treatment giving priority to novel medicines (bedaquiline, delamanid) and increased the importance of linezolid and clofazimine in the core medicines for MDR-TB treatment, which represented a significant price increase over older no-longer-recommended treatment regimens. MDR-TB patients must receive individually tailored treatment consisting of at least five medicines for different durations. ${ }^{16}$ Through the Global Drug Facility (GDF), the estimated treatment cost of longer individualized treatment regimens could reach at least US\$2000 for people who need at least 18 months of treatment with one novel therapy, representing a $50 \%$ price increase over previous standard treatment. For people who might need two novel therapies for 20 months, the increase of the treatment cost could reach $500 \%$, with one of the treatment regimen cost at around 
US\$ 9000. ${ }^{17}$ As a GDF non-eligible country, China has to negotiate the price by itself. Although the national price negotiation in China had the prices reduced at an average of more than $60 \%$ in $2019,{ }^{18}$ the national TB program would not be affordable for even if all the earmarked government funds for TB are to be shifted to MDR-TB, as the prices of the two novel therapies and other core medicines of the WHO newly recommended treatment regimens were still too high, and made the treatment cost for 6-12 month linezolid longer treatment regimens still reached US\$30,000 to US\$38,000 in China.

\section{Insecure Safety-Net for the Patient in Financial Hardship}

Under the basic health insurance system, patients are entitled to different benefits packages depending on their ability to pay. Patients have to pay a deductible (several values up to US\$ 250) and $10-30 \%$ patient co-payment OOP before getting $50-70 \%$ of the remaining expenditure reimbursed by the insurance. There are vast discrepancies of risk protection among patients entitled to different benefits packages. According to a 2012 survey conducted in 67 cities across 24 provinces of China, the median reimbursement rate of the outpatient expenditures of MDR-TB patients enrolled in the rural resident, urban resident, and urban employee programs were $60 \%, 60 \%$ and $80 \%$ $(p<0.05)$, that for inpatient expenditures were $70 \%, 70 \%$ and $80 \%(p<0.05){ }^{19,20}$

Wang et al and Yang et $\mathrm{al}^{21,22}$ found that the worse the economic status of the patient, the more likely they were to get TB. However, the risk protection yet built a safetynet for access to the needed treatment in China for the patients with weak ability to pay, especially the patients in financial hardship. A Ministry of Health commissioned survey about the economic burden of TB patients in six provinces in 2017 found that the average expenditure was US\$ 2400 and 16,000 for TB and MDR-TB patients; the patient OOP expenditure share was $68.0 \%$ and over $70.0 \%$. Their average direct medical expenditure was about US\$1900, (Unit of currency was transformed into US\$, US\$ $1=\mathrm{CNY} 7$ ). Among which the patient OOP expenditure share was $59.0 \%$. 39.9\% of TB patients complained about the negative financial impact on their families, among which $15.6 \%$ complained severe negative financial impact and $95.8 \%$ of their families encountered catastrophic expenditure. ${ }^{23}$

\section{Lack of Supportive Policies for Evidence- Based off-Label Use}

Except for the expensive novel medicines, most of the WHO recommended old anti-MDR-TB drugs are not covered by the Chinese basic health insurance, as they lack MDR-TB indication, and can only be used as off-label for MDR-TB. ${ }^{24}$ The exclusion of these antimicrobials by insurance further intensified the financial barrier of the MDR-TB patients, especially those in financial hardship and entitled to the weak benefits packages.

Off-label use of medicines has been the common practices globally in many therapeutic areas like oncology, paediatrics and the emerging diseases, which represent unmet medical needs. ${ }^{25}$ However, there is no legislative framework for the off-label use of medicines in China. On the contrary, the Drug Administration Law, the Infringement Liability Law and the Practitioner Law are all not in favour of evidence-based offlabel use of medicines. Although some health facilities develop internal regulations, doctors might be sued by the patient in case of dispute when prescribing off-label drugs. ${ }^{26,27}$ With the absence of legislative back-up for the evidence-based off-label use, these core antimicrobials are reimbursed by the insurance only for their first indication rather than MDR-TB. NRA approval is the only formal back-up for insurance coverage rather than expert consensus and evidence generated by the globally recognized academic organizations.

\section{No Incentive for the Investment in Repurposed Medicines}

To make the off-label use for MDR-TB treatment "legally", one solution would be to have the pharmaceutical company conducting the clinical study to get NRA approval. However, apart from the patent protection, there is no regulatory incentive in China for investment in the costly clinical study (US\$ 1 million) to extend the labelling of an established product, even for the communicable diseases with critical public health significance like MDR-TB. Moreover, it is more challenging for the off-patent products because patent claims for the secondary use often offer weaker protection compared to the primary compound patent protection, so the risk of freeriding by the competitors is high. ${ }^{28}$ It is challenging to enforce the patent protection for the newly added indication of an off-patent compound in the real world. Considering that getting NRA approval may not secure the return-on-investment and even may not be able to get the investment recovered, pharmaceutical companies often choose not to invest in the new therapeutic indication of an established product after 
the expiry of its primary patent protection. ${ }^{29,30}$ Unfortunately, most of the off-label used antimicrobials for MDR-TB are off-patent.

In addition to patent protection, regulatory exclusivities like test data protection may create an alternative incentive for the investment in the clinical study for indication expansion of an established product. Such regulatory exclusivities restrict others to use the clinical test data generated by the investors, which offer a certain degree of protection to the investors. However, it still does not resolve the enforcement concerns of the exclusivity of the established product after the expiry of its primary patent protection.

\section{Strategies to Promote Access to Affordable MDR-TB Medication Appropriate Financing Mechanism for Expensive Therapy}

The 2019 national price negotiation made a significant reduction in the prices of the novel medicines, including the WHO recommended two novel therapies for MDR-TB. However, they may still bring huge financial risks to the basic health insurance program with the extraordinarily high price, which calls for further price reduction through the continued price negotiations based on cost-effective evidence and the international reference price. Other strategies like generic competition are also needed to reduce the prices of not only the novel medicines but also all the national guide recommended core medicines for MDRTB. Appreciated to the recent national strategy of China to strengthen the research and development $(R \& D)$ capacity of the local pharmaceutical industry, there has been an increasing number of local firms conducted clinical studies of the generics. However, the earliest patent expiry date of the two novel medicines is $2023,{ }^{31}$ which meant that the generic competition would not be available in the coming 3 years. Unless the pharmaceutical corporations reduce their prices substantially, people will continue to suffer without access to safer and more effective treatment.

In addition to the novel therapies, the financial barrier of MDR-TB medication is also attributable to the recommended core antimicrobials used off-label for MDR-TB and not covered by the insurance program. Globally, there are debates about the coverage of off-label used medicines by health insurance. Most developed countries support the insurance coverage of off-label used medicines to some extent. German Federal Social Court stated in a first ruling in 2002 that, social health insurance should cover the effective treatment of severe health conditions supported by the existing evidence and not feasible for other treatment options, coverage denials restrict patients from receiving medically necessary therapies that represent the best available treatment options. ${ }^{32}$ The US common position is that doctors' treatment decisions should be in line with the patients' clinical needs, and support informed decision making that promotes third-party reimbursement for FDAapproved drug products appropriately prescribed for unlabeled uses. Off-label use of medicines may be justified to the Centers for Medicare and Medicaid Services by the authoritative drug information books. Phase III clinical trial data, the world top peer-reviewed medical journals, peer-reviewed textbooks and consensus guidelines, or by petitioning and obtaining approval from a local fiscal intermediary are also referenced. ${ }^{33}$ A growing number of insurance carriers have been encouraging the use of the authoritative drug compendia, peer-reviewed literature, and consultation with experts in research and clinical practice to make specific coverage decisions. The Chinese basic health insurance program should consider following the above practices.

\section{Safety-Net for the Patients in Financial Hardship}

China claimed to achieve universal basic health insurance coverage in the early 2010s. However, there is still a long way to achieve universal health coverage (UHC), which needs to build a safety-net for the patient in financial hardship. Although such a safety-net is yet built across the country, some areas have piloted some innovative strategies to construct the UHC for MDR-TB patients like the China-Gates pilots, which should be expanded across the country in terms of a government dominated, multi-sourced financing and coordination mechanism for the full course of diagnosis, treatment and management of MDR-TB, the "one-stop" settlement approach which integrated the reimbursement of the basic health insurance and the catastrophic health insurance, as well as the Health Financial Aid for the patients in financial hardship. It is critical to improve the benefits packages for patients enrolled in either the formal employee and the resident programs, remove the deductibles and the pre-reimbursement patient co-payment, keep the reimbursement rate at $70 \%$ or above, cover both outpatient and inpatient care and the services across the full course of treatment (including follow-up visits and tests and adversedrug-reactions). The coordination among the local finance, health insurance, and the health authorities is essential for the success of the pilots. As summarized in Figure 1, the earmarked 


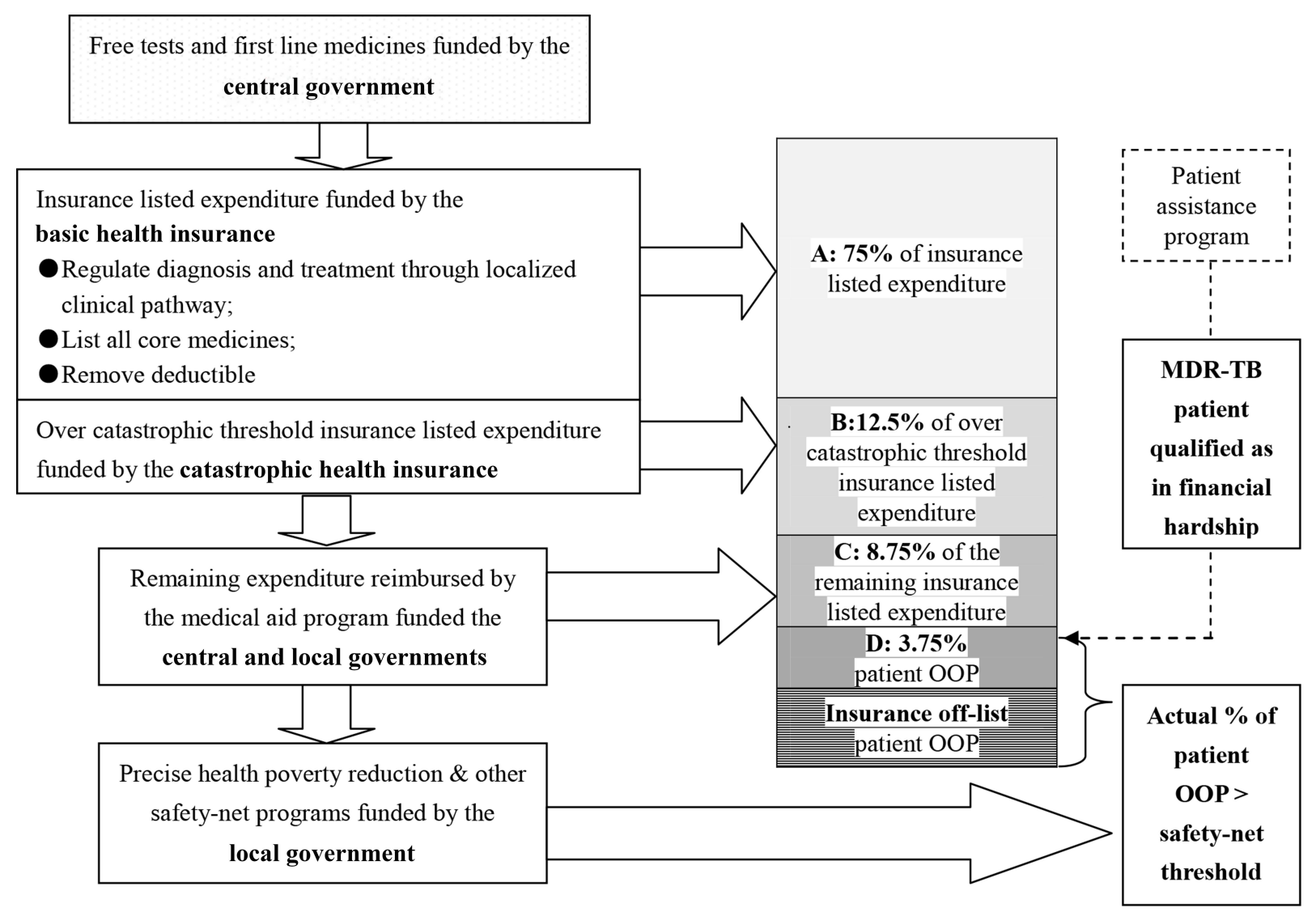

Figure I Coordinated and multi-sourced financing model and comprehensive approaches to relieve the financial burden of TB patient piloted in China.

Notes: $A=75 \%$ insurance listed expenditure reimbursed by the basic health insurance; $B=12.5 \%(25 \% \times 50 \%)$ over catastrophic threshold insurance listed expenditure reimbursed by the catastrophic health insurance; $C=8.75 \%(12,5 \% \times 70 \%)$ of the remaining insurance listed expenditure after catastrophic health insurance reimbursement covered by the medical aid program for patient in financial hardship as defined by the civil affairs department; $D=3.75 \%(12.5 \% \times 30 \%)$ of the remaining insurance listed expenditure after catastrophic health insurance reimbursement + insurance off-list expenditure paid by patient OOP. In case that the actual proportion of OOP > safety-net threshold (due to inflated insurance off-list expenditure), the précised health poverty reduction and other safety-net programs funded by the local government offer further aids. In some areas, patient assistance program funded by the civil society or the pharmaceutical company offer supplementary aids to the MDR-TB patient in financial hardship as defined by the civil affairs department. Bold texts indicate funding from different sources. Data from Wang $L$ and $C h e n g$. Theory and practice of financing and provider payment reform of tuberculosis. Beijing: People's Health Publishing House; 2019. Chinese. ${ }^{19}$

Abbreviations: OOP, out-of-pocket; MDR-TB, multidrug-resistant tuberculosis.

central government budget covered the free tests and first line medicines; the basic health insurance offered a reimbursement of $75 \%$ of the listed expenditure. On this base, patients might get $50 \%$ of the over catastrophic threshold insurance listed expenditure reimbursed by the catastrophic health insurance program. The safety-net was built to have the medical aid program to further cover $70 \%$ of the remaining insurance listed expenditure after catastrophic health insurance reimbursement for the patient in financial hardship as defined by the civil affairs department. In case that the actual proportion of OOP payment is over $30 \%$ for susceptive TB patient and over $10 \%$ for MDRTB patient (safety-net threshold) due to inflated insurance offlist expenditure (totally paid OOP by patient), the précised health poverty reduction and other safety-net programs funded by the local government offered further aids. Some areas also set up patient assistance program funded by the civil society or the pharmaceutical company to offer supplementary aids following the publicly funded aids to the MDR-TB patient in financial hardship as defined by the civil affairs department. ${ }^{14,19}$

To contain the expenditure and to use the funds more cost-effectively, the China-Gates pilots also implemented the provider payment reforms. The reforms shifted the payment method from the traditional fee-for-service to a case-based model, which covered the full course of diagnosis, test, treatment and management. Localized clinical pathways were also formulated, which included the locally adapted treatment options for MDR-TB and considered the severity, complications and co-existing diseases. ${ }^{19}$

\section{Backup of Evidence-Based off-Label Use}

Many industrialized countries establish the legal framework for evidence-based off-label use of medicines. One 
valuable model would be the French Temporary Recommendations for Use (TRU) strategy to ensure safe and effective off-label use of medicines. The NRA sets up a committee to systematically analyze and generate evidence, monitor and disseminate unbiased information, and establish a national database for off-label use of medicines. The NRA also mobilizes all related authorities to coordinate the regulation of off-label use of medicines, including providing funding to the pharmaceutical industry and research institutes to monitor off-label use of medicines and offering compensation to patients suffered from damages due to off-label use of medicines. ${ }^{34}$ Such a legal framework and operational mechanism not only offers backup for the prescribers but also helps to generate evidence for scientific use and potential formal regulatory approvals. In addition, a TRU decision is issued a single time for any given drug and may result in a right to reimbursement for the drug for the designated indication. The development of the TRU is a major step forward for public health and paves the way for tighter control of offlabel prescribing, it also hastens the conduct of clinical research and fosters the development of new indications for specific subgroups of patients or rare diseases. ${ }^{35}$ The Chinese NRA is expected to play a similar role with the coordination of the health and the health security authorities. More challenging is to regulate the pharmaceutical firms for the patient follow-up, the collection of efficacy and safety information, the real conditions of use, and the schedule for reporting data.

\section{Regulatory Incentives for Investment in Repurposed Medicines}

Discovering new use of existing medicines offers great promise for quickly bringing new therapeutic options. The lag between discovery and availability of the new options often 10 years or longer, which could be shortened by repurposing the marketed medicines, and could offer safe, timely, and affordable new treatment options particularly useful in the areas with high unmet needs. ${ }^{36}$ Questions arise on how the new uses will be translated into clinical practice, especially in the case of the offpatent products with expired exclusive rights. There are various legal approaches to get new therapeutic indications approved and to bring them on-label. One route is that the pharmaceutical companies go through marketing authorization procedures, which may entail high costs and need strong incentives. To create such incentives, many regulatory authorities including the US Food and Drug Administration (FDA) and the European Medicines Agency developed policies to grant additional market exclusivity to the market authorization holders (MAHs) for specific conditions. ${ }^{30}$ In the US, new clinical investigation and orphan drug designation can gain 3-7 years of market exclusivity, as well as tax breaks, waivers of FDA fees and other incentives. ${ }^{37}$ The recent orphan drug designation of the experimental drug remdesivir for COVID-19 by the US FDA is a real case of the regulatory incentive. ${ }^{38}$ In the EU, MAHs can be granted with one additional year of market protection if one or more new therapeutic indications bringing significant benefit in comparison with existing therapies are registered within the first eight years after initial approval. Considering that the generics may be available on the market only after the patent expires (20 years since the patent granted), the new therapeutic indication for generics and even for the patented medicines 8 years after the registration cannot benefit from the additional protection. ${ }^{30,39}$ Besides, the regulatory exclusivities relevant to the development of new use in the EU also grant a non-cumulative period of one-year data exclusivity for the new indication of a well-established substance if significant pre-clinical or clinical studies were carried out concerning the new indication. However, a report about pharmaceutical incentives and rewards in the EU showed that this incentive had never been granted for any centrally approved substance. ${ }^{40}$

In the Chinese setting, the 2017 Medicines Registration Regulation committed to grant data protection to the medicines for rare diseases (Article 116). ${ }^{41}$ Detailed regulations about data protection were proposed in the Implementation Regulation of Medicines Test Data Protection (2018 draft) in which 6-year data protection was proposed to be granted to the clinical trial data of medicines for rare diseases since its first approval in China (Article 6). ${ }^{42}$ The 2019 Regulations for the Implementation of the Drug Administration Law clearly defined that the self-obtained undisclosed test data of novel medicines will be granted 6-year protection (Article 34). ${ }^{43}$ All these regulations are expected to create incentives for $R \& D$ of novel products, but there is no specific description for repurposed off-patent medicines. If MDR-TB can be designated as a rare disease, the MAH of the repurposed medicines for MDR-TB would be entitled to 6-year data protection. However, the enforcement of such regulatory exclusivity may still face difficulties in the real world.

Apart from the pharmaceutical industry, the public initiative may be another approach to achieve the clinical adoption 
of a repurposed product. In 2008, the National Major New Drug R\&D Project and the National Prevention and Treatment Project for Major Communicable Diseases were established based on the National Mid- to Long-term Science \& Technology Development Planning Framework (20062020). ${ }^{44,45}$ The central government of China invested about US\$ 2.3 billion in the R\&D of novel medicines from 2008 to 2018. By the end of 2018, 35 novel medicines were locally developed and marketed. ${ }^{46}$ However, this public-funded project focused very much on screening new chemical and biochemical entities rather than the new use of the established products, even for the significant communicable disease with critical public health implications like MDR-TB. It would be ideal to have the repurposing study for MDR-TB included in this Project. Having the high costs, infrastructure, expertise and resources to fulfil the requirements for obtaining and maintaining a marketing authorization funded by the public funds will result in more affordable repurposed medicines. Such an indispensable role of the public funding has been very much favoured during the current corona-virus epidemics in China, to which a massive amount of public funding has been allocated for the screening of the established products for the development of anti-corona-viral regimens. ${ }^{47}$

\section{Conclusions}

In the short run, we would suggest the Chinese health authority to update the national guideline for MDR-TB treatment as soon as possible, listing all the recommended medicines in the National Essential Medicines List, and enabling them benefited from the supportive policies for the rare diseases. The National Major New Drug R\&D Project should support the $R \& D$ of both novel medicines and the repurposed off-patent medicines. The national health security authority should continue the price negotiation of those expensive medicines based on cost-effective evidence and international reference price, and cover all the national treatment guideline recommended medicines for MDR-TB.

In the long run, we would suggest the government to follow the French model, developing the legislative framework for the TRU of medicines and setting up a coordinative mechanism among the finance, health, health security, and drug regulatory authorities at all levels, as well as the healthcare providers, the academics and the civil societies, and setting up a task force to address the issue of access barriers of MDR-TB treatment in China. The strategies would include a multi-sourced financing model with the regularly increased government subsidy for the MDR-TB, most importantly is a safety-net for the high-cost and low-ability-to-pay patient. The timely and coordinated update of the national treatment guideline, the insurance coverage, and benefits packages for the health problems with significant social, economic and political implications is also expected.

\section{Acknowledgments}

The authors appreciate all the key informants who provided valuable supports and shared their insights with the study team.

\section{Disclosure}

All of the authors declare that there are no personal, commercial, political, and any other potential conflict interests related to the published paper.

\section{References}

1. World Health Organization. Rapid Communication: Key Changes to Treatment of Multidrug-and Rifampicin-resistant Tuberculosis (MDR/ $R R-T B)$. Geneva: World Health Organization; 2018.

2. World Health Organization. The Use of Bedaquiline in the Treatment of Multidrug-resistant Tuberculosis: Interim Policy Guidance. Geneva: World Health Organization; 2013.

3. Lange C, Aarnoutse RE, Alffenaar JWC, et al. Management of patients with multidrug-resistant tuberculosis. Int $J$ Tuberc Lung Dis. 2019;23(6):645-662. doi:10.5588/ijtld.18.0622

4. World Health Organization. Global Tuberculosis Report 2019. Available from: https://apps.who.int/iris/bitstream/handle/10665/ 329368/9789241565714-eng.pdf. Accessed 8 June 2020.

5. Lonnroth K, Glaziou P, Weil D, et al. Beyond UHC: monitoring health and social protection coverage in the context of tuberculosis care and prevention. PLoS Med. 2014;11(9):e1001693. doi:10.1371/ journal.pmed. 1001693

6. Richter LM, Lonnroth K, Desmond C, et al. Economic support to patients in HIV and TB grants in rounds 7 and 10 from the global fund to fight AIDS, tuberculosis and malaria. PLoS One. 2014;9(1): e86225. doi:10.1371/journal.pone. 0086225

7. Herbert N, Sharma V, Masham BS, et al. Concrete action now: UN high-level meeting on tuberculosis. Lancet Infect Dis. 2018;18 (7):709-710. doi:10.1016/S1473-3099(18)30171-3

8. World Health Organization. Tuberculosis country profiles. Available from: https://www.who.int/tb/country/data/profiles/en/. Accessed June 8, 2020.

9. Jiang J, Lucas H, Long Q, et al. The effect of an innovative financing and payment model for tuberculosis patients on health service utilization in China: evidence from Hubei Province of China. Int J Environ Res Public Health. 2019;16:14. doi:10.3390/ijerph16142494

10. Ren C, Lin H, Yang S. Epidemic characteristics of tuberculosis and multidrug-resistant strains and strategies. Chinese J Lung Dis. 2019;3 (269274):. doi:10.3877/cma.j.issn.1674-6902.2019.03.001

11. Long Q, Qu Y, Lucas H. Drug-resistant tuberculosis control in China: progress and challenges. Infect Dis Poverty. 2016;5:9. doi:10.1186/ s40249-016-0103-3

12. TB Prevention and Control Center of China CDC. The Comprehensive TB Control Model: Baseline Survey Report. in Chinese. Beijing: China CDC;2013.

13. Wang $\mathrm{L}$, Nunn $\mathrm{PP}, \mathrm{Xu} \mathrm{S}$ Achievements and Challenges in Tuberculosis Control in Modern China.1st Edition. Beijing: People's Medical Publishing House; 2016. (in Chinese)

14. Xin YJ, Xiang L, Jiang JN, et al. The impact of increased reimbursement rates under the new cooperative medical scheme on the financial burden of tuberculosis patients. Infect Dis Poverty. 2019;8(1):67. doi:10.1186/s40249-019-0575-z 
15. The Ministry of Human Resources and Social Security. The national reimbursement list of the basic health insurance (2017 edition). Available from: http://www.mohrss.gov.cn/SYrlzyhshbzb/dongtaixin wen/buneiyaowen/201702/t20170223_266787.html. Accessed June 6, 2020. (in Chinese)

16. World Health Organization. WHO consolidated guidelines on drugresistant tuberculosis treatment. Available from: https://apps.who.int/ iris/bitstream/handle/10665/311389/9789241550529-eng.pdf?ua=1. Accessed June 7, 2020.

17. Frontières MS. MSF warns high prices of tuberculosis drugs to restrict people's access to best drug-resistant TB treatment. (content current as of 2018-10-20). Available from: https://msf-seasia.org/ news/18360. Accessed June 8, 2020.

18. The National Healthcare Security Administration of China. The announcement of the inclusion of the novel medicines in the national basic health insurance medicines reimbursement list through the 2019 national medicines price negotiation. No. 65 document of 2019. (content current as of 2019-11-28). Available from: http://www.nhsa.gov.cn/art/ 2019/11/28/art_37_2050.html. Accessed June 8, 2020. (in Chinese)

19. Wang L, Cheng B. Theory and Practice of Financing and Provider Payment Reform of Tuberculosis. in Chinese. Beijing: People's Health Publishing House; 2019:p49.

20. Xu C, Ma W, Zhang C, et al. Analysis of the reimbursement of MDRTB expenditures by different basic health insurance programs in China. Chn J Prev Med. 2014;7:633-635. doi:10.16506/j.10096639.2014.07.012;

21. Wang G, Sleigh A, Zhou G, et al. Non-biologic risk factors of pulmonary tuberculosis among adults in Henan: a case-control study. Chin J Epidemiol. 2005;2:20-24.

22. Yang J, Liu M, Yang F, et al. Analysis of influencing factors of tuberculosis incidence using a two-level logistic regression model. Chin J Health Stat. 2009;4:374-376. (in Chinese)

23. China $\mathrm{CDC}$ and Peking University. Analysis results of the national survey of the economic burden of TB patients. Unpublished report. (in Chinese)

24. Sotgiu G, Pontali E, Migliori GB. Linezolid to treat MDR-/XDRtuberculosis: available evidence and future scenarios. Eur Respir $J$. 2015;45(1):25-29. doi:10.1183/09031936.00145014

25. Weda MHJ, Vervloet M, et al. Study on Off-Label Use of Medicinal Products in the European Union. Belgium, Brussels: European Union; 2017.

26. Sachs RE, Ginsburg PB, Goldman DP. Encouraging new uses for old drugs. JAMA. 2017;318(24):2421-2422. doi:10.1001/jama.2017.17535

27. Wu Q "Why do I treat a hospice cancer patient with Morphine?"the first case of prosecution of morphine related medical care. (content current as of 2017-09-24). Available from: http://www. china-pharmacy.com/2/232/26198.html. Accessed June 8, 2020. (in Chinese)

28. Guangdong Pharmaceutical Association. Expert's consensus on unregistered medicines use 2010. (content current as of 2015-01-22). Available from: http://sinopharmacy.com.cn/download/1.html. Accessed June 8, 2020. (in Chinese)

29. Langedijk J, Whitehead CJ, Slijkerman DS, et al. Extensions of indication throughout the drug product lifecycle: a quantitative analysis. Drug Discov Today. 2016;21(2):348-355. doi:10.1016/j.drudis.2015.11.009

30. Nayroles G, Frybourg S, Gabriel S, et al. Unlocking the potential of established products: toward new incentives rewarding innovation in Europe. J Mark Access Health Policy. 2017;5(1):1298190. doi:10.1080/20016689.2017.1298190

31. The Medicines Patent and License Database. MedsPaL. Available from: https://www.medspal.org/?country_name $\% 5 \mathrm{~B} \% 5 \mathrm{D}=$ China\&page $=1$. Accessed June 8, 2020.

32. Smieliauskas F, Sharma H, Hurley C, et al. State insurance mandates and off-label use of chemotherapy. Health Econ. 2018;27(1):e55e70. doi: $10.1002 /$ hec. 3537
33. Johnson PE Pharmaceutical reimbursement: an overview. $A m j$ Health-System Pharm. Suppl.2008;1:S4.:.

34. L'Agence nationale de sécurité du médicament et des produits de santé. Available from: https://www.ansm.sante.fr/L-ANSM/Uneagence-d-expertise/L-ANSM-agence-d-evaluation-d-expertise-et-dedecision/(offset) $/ 0$. Accessed June 8, 2020. (in French)

35. Emmerich J, Dumarcet N, Lorence A. France's new framework for regulating off-label drug use. $N$ Engl $J$ Med. 2012;367(14):12791281. doi:10.1056/NEJMp1208347

36. Verbaanderd C, Rooman I, Meheus L, et al. On-label or off-label? Overcoming regulatory and financial barriers to bring repurposed medicines to cancer patients. Front Pharmacol. 2019;10:1664. doi:10.3389/fphar.2019.01664

37. US FDA. Frequently asked questions on patents and exclusivity. (content current as of 2018-05-02). Available from: https://www.fda.gov/drugs/ development-approval-process-drugs/frequently- asked-questions-patentsand-exclusivity\#howlongexclusivity. Accessed June 8, 2020.

38. Consumer News and Business Channel. Biontech and Pharma. Gilead's potential coronavirus treatment gets FDA's orphan drug label. (content current as of 2020-03-24). Available from: https:// www.cnbc.com/2020/03/24/gileads-potential-coronavirus-treatmentgets-fdas-orphan-drug-label.html. Accessed June 8, 2020.

39. European Commission. Enterprise and industry directorate-general. Guidance on a new therapeutic indication for a well-established substance. Belgium, Brussels: european Commission; 2007. Available from: https:// ec.europa.eu/health/sites/health/files/files/eudralex/vol-2/c/10\%2520_5_\% 2520guideline_11-2007_en.pdf. Accessed June 8, 2020.

40. Copenhagen Economics. Directorate-general for internal market, industry, entrepreneurship and SMEs. Directorate $\mathrm{f}$ - innovation and advanced manufacturing. Unit GROW-F.5 - intellectual property and fight against counterfeiting. Study on the economic impact of supplementary protection certificates, pharmaceutical incentives and rewards in Europe. Belgium, Brussels: European Commission; 2018. Available from: https://ec.europa. $\mathrm{eu} /$ health/sites/health/files/human-use/docs/pharmaceuticals_incentives_ study_en.pdf. Accessed June 8, 2020.

41. National Medical Products Administration. The general office of national medical products administration publicly solicited opinions on medicines registration regulation (revised version). (content current as of 2017-10-23). Available from: http://www.nmpa.gov.cn/ WS04/CL2095/229342.html. Accessed June 8, 2020. (in Chinese)

42. National Medical Products Administration. The general office of National Medical Products Administration publicly solicited opinions on Implementation Regulation of Medicines Test Data Protection (2018 draft, call for comments). (content current as of 2018-04-25). Available from: http://www/nmpa/gov.cn/WS04/CL2051/227856. html. Accessed 8 June 2020 (in Chinese)

43. China's open law platform. The 2019 regulations for the implementation of the drug administration law. Available from: http://open.pku law.cn/FullText/ViewFullText?library=chl\&gid=330916\& match= Exact. Accessed June 8, 2020. (in Chinese)

44. National Science and Technology Major Project. Major projects. Available from: http://www.nmp.gov.cn/zxjs/. Accessed June 8, 2020. (in Chinese)

45. Sun YMX, Li P, et al. National science and technology major projects for new drug development: A new engine for China's drug innovation system. Chin J New Drug. 2013;8:868-872. (in Chinese)

46. G S. Progress of the 2018 national major new medicines developments project and forecast of the 13th five-year plan. China Biotechnol. 2019;2:3-12. (in Chinese)

47. The Ministry of Science and Technology. Having science and technology playing a key role in defeating the epidemics. Seek Truth 2020;6. (content current as of 2020-03-16). Available from: http://www.qstheory.cn/dukan/ qs/2014/2020-03/16/c_1125710486.htm. Accessed June 8, 2020. (in Chinese) 


\section{Publish your work in this journal}

Infection and Drug Resistance is an international, peer-reviewed openaccess journal that focuses on the optimal treatment of infection (bacterial, fungal and viral) and the development and institution of preventive strategies to minimize the development and spread of resistance. The journal is specifically concerned with the epidemiology of antibiotic resistance and the mechanisms of resistance development and diffusion in both hospitals and the community. The manuscript management system is completely online and includes a very quick and fair peerreview system, which is all easy to use. Visit http://www.dovepress.com/ testimonials.php to read real quotes from published authors. 